\title{
Image-Based Fire Detection Using Neural Networks
}

\author{
Wen-Bing Horng Jian-Wen Peng \\ Department of Computer Science and Information Engineering \\ Tamkang University, Taipei, Taiwan 25137, ROC \\ E-mail: \{horng@mail.tku.edu.tw, pchw8598@gmail.com\}
}

\begin{abstract}
An image-based fire detection method using neural networks is proposed in this paper. First, flame color features, based on the HSI color model, are trained by a backpropagation neural network for flame recognition. Then, based on the learned flame color features, regions with fire-like colors are roughly separated from an image. Besides segmenting flame regions, background objects with similar fire colors or resulted from the reflection of fire flames are also separated from the image. In order to get rid of these spurious fire-like regions, the image difference method and the invented color masking technique are applied. Finally, a compact method is devised to estimate the burning degree of fire flames so that users could be informed with a proper warning alarm. The proposed system can achieve $96.47 \%$ fire detection rate on average.
\end{abstract}

Keywords: burning degree estimation, color masking, fire flame detection, neural network.

\section{Introduction}

With the gradual popularity of installation of visual surveillance systems in the past decades, fire flame detection has become a very important issue because it is closely related to people's safety and property. Today, most frequently used flame detection techniques are usually based on particle sampling, temperature sampling, and air transparency testing, in addition to the traditional ultraviolet and infrared flame detectors [1]. However, most of these detectors suffer from some severe problems. They require a close proximity to the flame. In addition, they are not always reliable, because they do not always detect the combustion itself. Instead, they detect the byproducts of combustion, which may be produced in other ways. Therefore, they usually result in higher false rates. Furthermore, all of these methods seldom provide additional descriptive information about flame location, size, burning degree, and so on.

Recently many research works on visual fire flame detection have been proposed. This is because images can provide more confident information.
Healey et al. [6] presented a fire detection system using color video input for a pre-allocated view on some ideal conditions. Noda and Ueda [10] used gray-scale images obtained from cameras to detect fire in tunnels. Yamagishi and Yamaguchi [12] also proposed a fire flame detection algorithm for color images based on the HSV color space and employed an artificial neural network to determine fire from Fourier transform of fire contours. Foo [3] presented methods for detecting fire in aircraft dry bays and engine compartments from gray-scale images. All of the above methods require a stationary camera. In addition, most of them are designed for a specific environment. Very recently, Phillips et al. [11] proposed a sophisticated method for recognizing flames in color video. However, their method does not consider the temporal variation of flames. Dedeoglu et al. [2] utilized wavelet transform to analyze the property of fire of high turbulence both on time and space. Liu et al. [9] employed Gaussian distribution to model the fire colors and Fourier transform to describe the fire contours. However, the above two are limited by regular convex flames and erroneous detection on small flames and fire-like areas.

Horng et. al. [7] also proposed an image-based real-time fire flame detection based on color analysis tried to get rid of the above drawbacks. However, in the fire areas extraction part, the HSI attributes of fires were determined by observation from test images, which might lead to be incomplete to describe fire flame color model. Therefore, in order to capture fire flame more accuracy, a backpropagation neural network is used to learn the fire color features in this paper so that fire detection can be greatly improved.

\section{Building a Flame Feature Model}

In this research, a neural network [5] is employed to build the flame color feature model (FCFM) because neural nets have the learning capability. Flame images of training samples are fed into the neural network to learn the color features of fire flames according to the HSI color model. In this paper, a four-layer fully connected backpropagation neural network is used for learning, as shown in Fig. 1. This net is a $3 \times 15 \times 8 \times 1$ network. The log-sigmoid function is used as the 
transfer function. The network input data are $H, S, I$ components of each pixel in an image, and it outputs the result which determines whether the pixel is a fire pixel or not with a pre-defined threshold 0.5. The initial weights of the network are randomly generated.

Since the employed backpropagation neural network is supervised, it requires a target output for each pixel. Therefore, for each training image, a mask image for the fire regions is created manually for neural network learning. As shown in Fig. 2, a mask image is a black and white image, in which the black areas correspond to fire flame regions and the white areas correspond to non-fire flame regions. In the learning stage, the network converges with more than $95 \%$ recall rate after three epochs. Fig. 3 shows the results of a test image using the trained neural network.

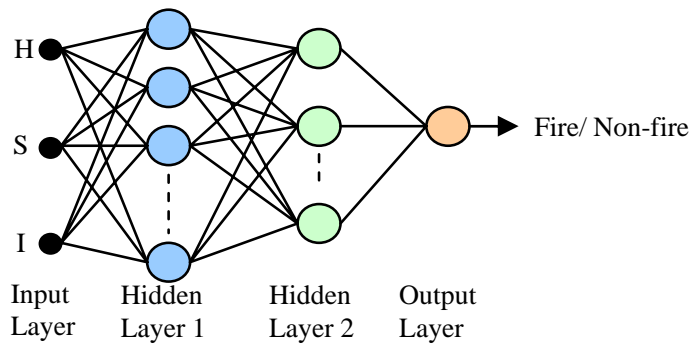

Fig. 1: The neural network for fire flame segmentation.
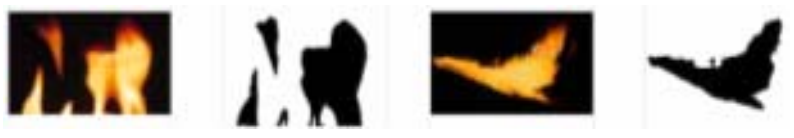

Fig. 2: Two training samples and their mask images, in which black areas correspond to fire areas of training samples.

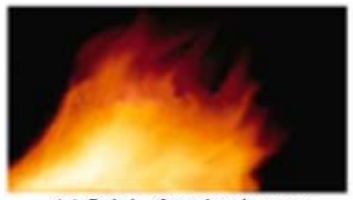

(a) Original testing image

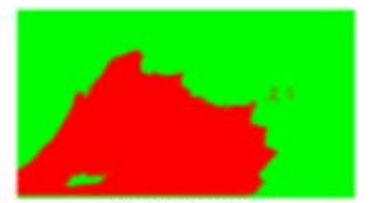

(b) Testing result
Fig. 3: Testing result. (a) Original testing image. (b) The green areas stand for background and the red areas indicate the detected fire areas.

\section{The Flame Detection Method}

The proposed fire flame detection method can be divided into three major phases: (1) flames segmentation using the trained neural network, (2) removal of spurious fire-like regions, and (3) estimation of the burning degree of fire flames.

In the first phase, based on the FCFM built by the neural network, fire-like color areas are roughly separated from an input image frame. Then, spurious regions caused by fire reflection or with similar fire-like colors are removed by the image difference and the color masking techniques [7]. Finally, the burning degree of fire is roughly estimated in the last phase to provide users a proper fire alarm.

\subsection{Fire Areas Segmentation}

The three components $(H, S, I)$ of each pixel of an image are sequentially input into the trained neural network and fire flame areas are extracted. For each pixel in the image $f(x, y)$, if the color of the pixel does not belong to the detected flame areas, then set the pixel color to black, otherwise, keep the original pixel color unchanged. The result image, $g(x, y)$, after performing the above fire flame segmentation procedure, can be represented as Eq. (1). The segmentation result is illustrated in Fig. 4.

$$
g(x, y)= \begin{cases}\text { black, } & \text { if } f(x, y) \notin F C F M \\ f(x, y), & \text { otherwise. }\end{cases}
$$

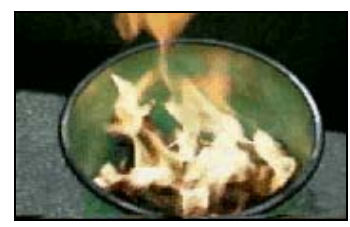

(a)

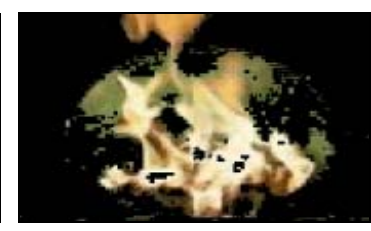

(b)
Fig. 4: (a) Original Image. (b) Flame segmentation by neural network.

\subsection{Removal of Spurious Fires}

There are two spurious fires need to be considered: (1) Reflection of fire flames, called color shift. (2) Nonfire objects with similar fire-colors.

For the first situation, it can be removed by filtering out the pixels with lower intensity and lower saturation than the fire source pixels. For the second situation, if the color of a background object is similar to fire flames, then the image difference method might be used to remove such an object. One special situation occurs when the color shift phenomena happens too fast so that the reflective color at the same place of two consecutive images differs quite a lot. After performing color segmentation and image difference, the resulting image still contains some apparent fire-color residues in the non-flame regions. In this case, color masking can be applied to solve such a problem [7]. By using the mask color, denoted by mask, for color masking, Eq. (1) for fire segmentation should be modified as Eq. (2), the result is shown in Fig. 5.

$$
g^{\prime}(x, y)= \begin{cases}\text { mask, } & \text { if } f(x, y) \notin F C F M \\ f(x, y), & \text { otherwise. }\end{cases}
$$




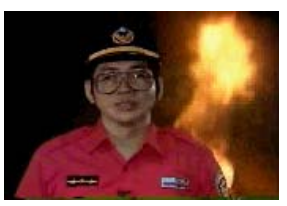

(a)

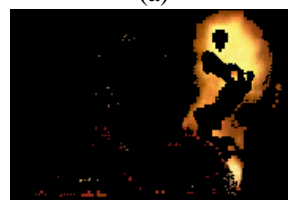

(c)

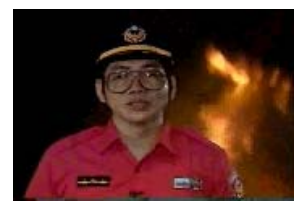

(b)

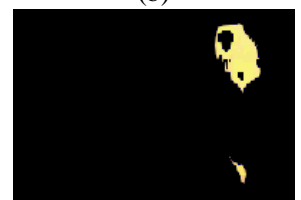

(d)
Fig. 5: (a) and (b) Two consecutive fire images; (c) Result after image difference using original color separation on (a) and (b), more color residues of red clothes around the fireman; (d) Applied the color masking and the residues on (c) are removed.

\subsection{Estimation of the Burning Degree of Fire Flames}

The difference of two consecutive $g^{\prime}(x, y)$ is called a contour image, $q(x, y)$. When the combustion is getting more violent, flame contours change their shapes more rapidly. Therefore, the variation of flame contour is used to estimate the burning degree of fire flames. The contour difference image, denoted as $d(x, y)$ will track the variation in two consecutive contour images, $q_{i}(x, y)$ and $q_{j}(x, y)$. In $d(x, y)$, a pixel is assigned to be black if it is a black pixel in both consecutive contour images; otherwise, the pixel is set to white. The image $d(x, y)$ is defined as Eq. (3).

$$
d(x, y)= \begin{cases}\text { black }, & \text { if } q_{i}(x, y)=q_{j}(x, y)=\text { black } \\ \text { white }, & \text { otherwise }\end{cases}
$$

After obtaining the contour difference image, the number of white pixels on it could be used as a measure for the burning degree of the fire flames. Define the white pixel ratio, $r_{w}$, of a contour difference image, $d(x, y)$, to be Eq. (4)

$$
r_{w}=\frac{n_{w}}{n}
$$

where $n_{w}$ is the number of white pixels, and $n$ is the total number of pixels in the image $d(x, y)$. The higher the white pixel ratio is, the more violently the fires burn.

In order to alleviate the problem of focal length, a small fraction (for example, 0.03), $s$, is given by the user according to the situation of the input video. Two threshold values, $t_{1}$ and $t_{2}$, are defined such that $t_{i}=i \times$ $s$, for $i=1$ and 2 . These threshold values serve as references for estimating the burning degree of fire flames. In this research, four estimated different burning degrees as used, as shown in Eq. (5). The estimation results are shown in Fig. 6.

$$
\text { burning degree }= \begin{cases}\text { no fire, } & \text { if } r_{w}=0 \\ \text { small, } & \text { if } 0<r_{w} \leq t_{1} \\ \text { medium, } & \text { if } t_{1}<r_{w} \leq t_{2} \\ \text { big, } & \text { if } r_{w}>t_{2}\end{cases}
$$

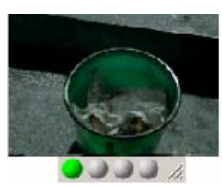

(a) no fire

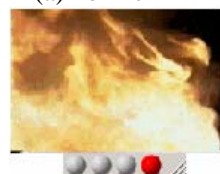

(d) big fire

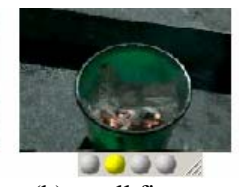

(b) small fire

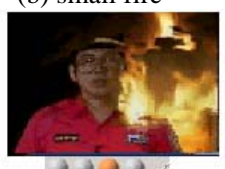

(e) medium fire

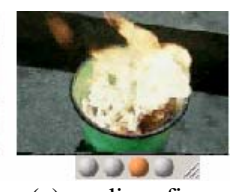

(c) medium fire

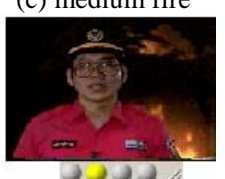

(f) small fire
Fig. 6: Four alert signs indicating different burning degrees of fire flames (green: no fire, yellow: small, orange: medium, red: big).

\section{Experimental Results}

The proposed fire flame detection method is tested with eleven fire video clips for a variety of conditions, including daytime and nighttime, indoor and outdoor. The experimental results of the proposed flame detection method are shown in Table I.

TABLE I

EXPERIMENTAL RESULTS OF FIRE FLAME DETECTION

\begin{tabular}{crrrrrl}
\hline \hline video & \multicolumn{1}{c}{$n_{f}$} & \multicolumn{1}{c}{$n_{i}$} & \multicolumn{1}{l}{$f_{-}$} & \multicolumn{1}{c}{$r_{d}(\%)$} & \multicolumn{1}{c}{ Description } \\
\hline 1 & 1189 & 875 & 2 & 22 & 97.98 & Fire in kitchen \\
2 & 674 & 575 & 5 & 22 & 95.99 & Fire and fireman \\
3 & 790 & 755 & 0 & 2 & 99.75 & Fire indoor \\
4 & 317 & 267 & 0 & 25 & 92.11 & Explosion \\
5 & 55 & 2 & 0 & 0 & 100.00 & Gun fire \\
6 & 733 & 733 & 0 & 0 & 100.00 & Spirit lamp in the daytime \\
7 & 558 & 558 & 66 & 11 & 86.20 & Spirit lamp with red clothes \\
8 & 791 & 791 & 0 & 0 & 100.00 & Spirit lamp in the nighttime \\
9 & 425 & 425 & 0 & 0 & 100.00 & Protesters \& gasoline bombs \\
10 & 759 & 601 & 0 & 87 & 88.54 & The burning vehicle \\
11 & 3648 & 3648 & 109 & 0 & 97.01 & Mountain fire \\
\hline total & 9939 & 9230 & 182 & 169 & $\mathbf{9 6 . 4 7}$ & \\
\hline \hline
\end{tabular}

The field $n_{f}$ is the total number of frames of a video clip. The field $n_{i}$ is the number of frames containing fire flames in the video. The field $f_{-}$is false negative, the field $f_{+}$is false positive. The field $r_{d}$, the detection rate of a video, is defined as $\mathrm{Eq}(6)$

$$
r_{d}=\frac{n_{c}}{n_{f}}
$$

where $n_{c}\left(=n_{f}-f_{-}-f_{+}\right)$is the number of correctly detected frames (including fire and non-fire frames). 
According to the experimental results as shown in Table I, the average detection rate can achieve more than $96.47 \%$. In video 2 , very small fire flames on the initial combustion are also detected, as shown in Fig. $7(a)$. Even for the nearly steady flames such as spirit lamps on videos 6 to 8, the system can still detect fire flames correctly, as illustrated in Figs. 7(b) and 7(c). Moreover, in videos 9 to 11 , the camera continuously shakes or the scenes are moving all the time, and all of these videos have very complex backgrounds, as shown in Figs. 8. However, the detection results still keep more than $90 \%$. In general, the experimental results are very encouraging and promising.

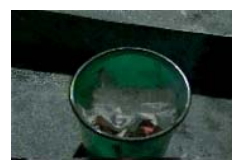

(a)

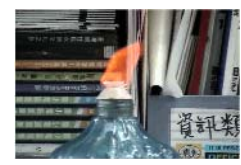

(b)

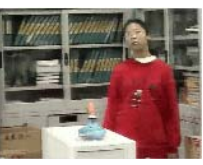

(c)
Fig. 7: (a) False negative detection on the initial combustion; (b) Spirit lamp with complex background; (c) Spirit lamp near a girl with red clothes.

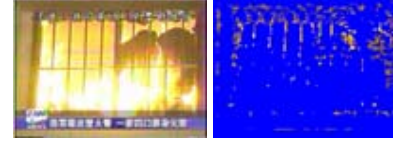

(a)

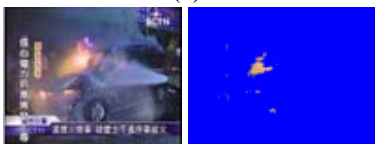

(c)

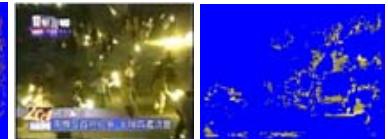

(b)

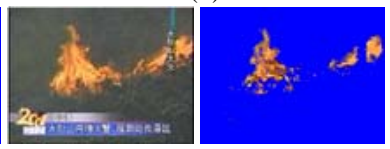

(d)
Fig. 8: Complex and shaking scenes, with their corresponding fire detection results.

\section{Conclusion and Discussion}

In this paper, an image-based fire flame detection method is proposed based on neural network learning and theory of chromatics. Several new ideas are presented in this new flame detection method. Fire flame color features are learned using neural networks. The color masking technique is proposed to cope with the color residue problem. In addition, a compact method is devised to estimate the burning degree of fire burning. The method is tested with eleven diverse video clips. The experimental results show that the method can achieve approximately $97 \%$ detection rate. In addition, the system can recognize fire flames on the initial combustion, and high ability to resist the camera shaking or scene moving.

In this research, though fire detection rate is quite high, fire detection on a 320x240 frame requires 1693 ms on a $1.7 \mathrm{GHz}$ CPU, which makes this system infeasible in real-time fire detection. In order to cope with the intensive computation power on neural net- work recall process for detecting fire pixel, recently the authors [8] have designed a very efficient method for detecting fire color, which greatly improved the fire detection performance. With this new method, fire detection on a frame of the same size only requires $1.85 \mathrm{~ms}$, which significantly makes the proposed fire detection system of practical use.

\section{References}

[1] B. C. Arrue, A. Ollero, and J. R. Martinez de Dios, "An Intelligent System for False Alarm Reduction in Infrared Forest-Fire Detection," IEEE Intelligent Systems, pp. 64-73, 2000.

[2] Y. Dedeoglu, B.U. Toreyin, U. Gudukbay, and A.E. Cetin, "Real-Time Fire and Flame Detection in Video," Proc. of 30th International Conference on Acoustics, Speech and Signal Processing, pp. 669-672, 2005.

[3] S.Y. Foo, "A Machine Vision Approach to Detect and Categorize Hydrocarbon Fires in Aircraft Dry Bays and Engine Compartments," IEEE Transaction on Industry Applications, vol. 36, pp. 549-466, 2000.

[4] R.C. Gonzales and R.E. Woods, Digital Image Processing, 2nd ed., Prentice-Hall, 2002.

[5] M.T. Hagan, H.B. Demuth, and M. Beale, Neural Network Design, PWS, 1996.

[6] G. Healey, D. Slater, T. Lin, B. Drda, A.D. Goedeke, "A System for Real-Time Fire Detection," Proc. of Computer Vision and Pattern Recognition Conference, pp. 605-606, 1993.

[7] W.B. Horng, J.W. Peng, and C.Y. Chen, “A New Image-Based Real-Time Fame Detection Method Using Color Analysis,” Proc. of International Conference on Networking, Sensing and Control, pp. 100-105, 2005.

[8] W.B. Horng and J.W. Peng, "A Fast Method in Detecting Fire Color," in preparation.

[9] C.B. Liu and N. Ahuja, "Vision Based Fire Detection," Proc. of the 17th International Conference in Pattern Recognition, vol. 4, pp. 673-676, 2004.

[10] S. Noda and K. Ueda, "Fire Detection in Tunnels Using an Image Processing Method,” Proc. of Conference on Vehicle Navigation and Information System, pp. 57-62, 1994.

[11] W. Phillips III, M. Shah, and N. da Vitoria Lobo, "Flame Recognition in Video," Pattern Recognition Letters, vol. 23, pp. 319-327, 2002.

[12] H. Yamagishi and J. Yamaguchi, "Fire Flame Detection Algorithm Using a Color Camera," Proc. of International Symposium on Micromechatronics and Human Science, pp. 255-260, 1999. 